\title{
On the representation of primes by cubic polynomials in two variables
}

\author{
D.R. Heath-Brown \\ Mathematical Institute, Oxford \\ B.Z. Moroz \\ Max-Planck-Institut für Mathematik, Bonn
}

\section{Introduction and statement of the main results}

The goal of this paper is to give a proof of the following theorem, which may be regarded as an instance of Schinzel's Hypothesis; it implies, in particular, a hypothesis introduced in a recent paper of the first author (see [2, Hypothesis 4]).

Theorem 1. Let $f_{0}(\vec{x})$ be a binary cubic form with integral rational coefficients irreducible in $\mathbb{Z}[\vec{x}]$. For $d \in \mathbb{Z}$ and $\vec{\gamma} \in \mathbb{Z}^{2}$, let the positive integer $\gamma_{0}$ be chosen so that $f(\vec{x})=\gamma_{0}^{-1} f_{0}(\vec{\gamma}+d \vec{x})$ is a primitive polynomial with integral rational coefficients. Suppose, moreover, that no prime divides $f(\vec{a})$ for every $\vec{a}$ in $\mathbb{Z}^{2}$. Then the set $f\left(\mathbb{Z}^{2}\right)$ contains infinitely many rational primes.

We shall use the technique developed in [3], and then applied in [4] to treat a general binary cubic form; consequently, the reader is advised to familiarize himself with the contents of those papers before studying the present one. As in $[3,4]$, we shall actually obtain an asymptotic formula for the relevant number of primes. Note that we cannot handle every binary cubic polynomial which splits over $\overline{\mathbb{Q}}$. In particular our methods do not suffice to treat the polynomial

$$
N_{\mathbb{Q}(\sqrt[3]{2}, \vec{x}) / \mathbb{Q}(\vec{x})}\left(x_{1}+x_{2} \sqrt[3]{2}+\sqrt[3]{4}\right)=x_{1}^{3}-6 x_{1} x_{2}+2 x_{2}^{3}+4, \vec{x}:=\left(x_{1}, x_{2}\right) .
$$

The reasons for this failure are subtle - the Type I sums can be handled satisfactorily, but not the Type II sums. 
In what follows, we shall retain the notational conventions introduced in $[4, \S 1]$. Let $\mathbb{C}, \mathbb{R}, \mathbb{Q}, \mathbb{Z}$, and $\mathbb{N}$ stand for the fields of complex, real, rational numbers, the ring of rational integers, and the set of positive rational integers, respectively. Let $k$ be a cubic number field; as in [4], let $\mathfrak{o}, \mathcal{I}, P$, and $\mathcal{P}$ denote respectively the ring of integers of $k$, the monoid of integral ideals in $k$, the set of rational primes, and the set of prime ideals in $\mathfrak{o}$. Given a sequence $D$ of rational integers and a sequence $\mathcal{D}$ of ideals in $\mathcal{I}$, let $D_{a}=\{c: c \in D, a \mid c\}$ and $\mathcal{D}_{\mathfrak{a}}=\{\mathfrak{c}: \mathfrak{c} \in \mathcal{D}, \mathfrak{a} \mid \mathfrak{c}\}$ for $a \in \mathbb{N}$ and $\mathfrak{a} \in \mathcal{I}$. In formulae, we shall sometimes use the abbreviation $(a, b):=$ h.c.f. $(a, b)$.

Let us fix a $\mathbb{Z}$-submodule $F$ of $\mathfrak{o}$ of rank 2 , an integer $d$ in $\mathbb{N}$, and a vector $\vec{\gamma}$ in $\mathbb{Z}^{2}$. As in [4], let $F=\left\{a_{1} \omega_{1}+a_{2} \omega_{2}: \vec{a} \in \mathbb{Z}^{2}\right\}$, so that $k=\mathbb{Q}\left(\theta_{0}\right)$ with $\theta_{0}:=\omega_{2} \omega_{1}^{-1}$. For $\vec{u} \in \mathbb{Z}^{2}$, we shall sometimes write $u=u_{1} \omega_{1}+u_{2} \omega_{2}$; in particular, $\gamma=\gamma_{1} \omega_{1}+\gamma_{2} \omega_{2}$. Let $\mathfrak{d}=\left(\gamma, d \omega_{1}, d \omega_{2}\right)$ be the ideal in $\mathfrak{o}$ generated by $d F$ and $\gamma$, and let

$$
f(\vec{x})=N_{k(\vec{x}) / \mathbb{Q}(\vec{x})}(\gamma+d x) N \mathfrak{d}^{-1} .
$$

Clearly, $f(\vec{x}) \in \mathbb{Z}[\vec{x}]$ and the polynomial $f(\vec{x})$ is irreducible in $\mathbb{Q}(\vec{x})$; moreover, it follows from the general theory (see, for instance, $[7$, chapter II]) that $f(\vec{x})$ is a primitive polynomial. Let $\varepsilon(f)$ denote the highest common factor of the integer values $\left\{f(\vec{a}): \vec{a} \in \mathbb{Z}^{2}\right\}$ of $f$. Write, for brevity, $\eta:=(\log X)^{-c}$, where $c$ is a suitable positive constant defined as in $[3,4]$, and consider the square

$$
I(X):=\left\{\vec{u}: \vec{u} \in \mathbb{R}^{2}, X<u_{1}, u_{2} \leq X(1+\eta)\right\} .
$$

Let

$$
\pi(f, X):=\#\{p: p \in P, p=f(\vec{a}), \vec{a} \in I(X)\} .
$$

Let $h_{f}:=d^{3} N\left(\mathfrak{d}^{-1}\left(\omega_{1}+\omega_{2}\right)\right)$, so that $f(\vec{a})=h_{f} X^{3}(1+O(\eta))$ for $\vec{a} \in I(X)$, cf. [4, Lemma 2.3]. Without loss of generality, it may be assumed that $h_{f}>0$. We can now state our main result.

Theorem 2. If $\varepsilon(f)=1$, then

$$
\pi(f, X)=\sigma_{1}(f) \frac{\eta^{2} X^{2}}{3 \log X}\left\{1+O\left((\log \log X)^{-1 / 6}\right)\right\}
$$

as $X \rightarrow \infty$, with $\sigma_{1}(f)>0$ given by (3.1).

Theorem 1 follows from Theorem 2 because the polynomial $f$ considered in Theorem 1 may be defined by an equation of the form (1.1).

Remark 1. In $[4, \S 2]$ it has been observed that $\varepsilon\left(f_{0}\right) \in\{1,2\}$ and that if $\varepsilon\left(f_{0}\right)=2$, then $f_{0}(\vec{x})=2 g(\vec{y})$, where $x_{1}=2 y_{1}, x_{2}=y_{2}$, and $g$ is a 
binary cubic form irreducible in $\mathbb{Z}[\vec{y}]$ with $\varepsilon(g)=1$. It will be shown here (see Lemma 2.4) that $\varepsilon(f) \in\{1,2,3,6\}$ and, moreover, $\varepsilon(f)=\varepsilon\left(f_{0}\right)$ if h.c.f.(6, $\mathrm{d})=1$.

As an application of our theorem we have the following results about rational points on cubic surfaces. We remark that unconditional results of the type given by Corollary 1.2 are few and far between.

Corollary 1.1 Suppose that $a_{1}, a_{2}, a_{3}, a_{4}, a_{5}$ are integers coprime to 3 , and assume that none of them is divisible by the square of any prime $p=2$ mod 3. Then if

$$
\sum_{i=1}^{5} a_{i} x_{i}^{3}=0
$$

has non-zero p-adic solutions for every $p$, it will have non-zero integral solutions, providing that the Selmer Conjecture holds.

Proof. This follows from [2, Theorem 4], since Hypothesis 4 introduced in [2] is a special case of Theorem 2 stated above.

Remark 2. For precise formulation of the Selmer (Parity) Conjecture see [2, Hypothesis 1].

Corollary 1.2 Let $a$ and $b$ be coprime rational integers satisfying one of the following congruence conditions:

$$
\text { a or } b= \pm 2 \text { or } \pm 3 \quad(\bmod 9) \text {, }
$$

or

$$
a= \pm b \quad(\bmod 9) .
$$

Then there is a nontrivial rational point on the surface

$$
x_{0}^{3}+2 x_{1}^{3}+a x_{2}^{3}+b x_{3}^{3}=0 .
$$

Proof. The result is trivial if $a / b$ is a rational cube. Otherwise the corollary will be an easy consequence of our theorem and a result of Satgé $[6$, Proposition 3.3]. The latter states that the curve

$$
x_{0}^{3}+2 x_{1}^{3}=p Z^{3}
$$

has a non-trivial rational point for any prime $p=2(\bmod 9)$. Under the hypotheses of the corollary we can find integers $a_{0}, b_{0}$ such that either $a a_{0}^{3}+$ $b b_{0}^{3}=2(\bmod 9)$, in the case of $(1.3)$, or $27 \mid a a_{0}^{3}+b b_{0}^{3}$ and $\left(a a_{0}^{3}+b b_{0}^{3}\right) / 27=2$ $(\bmod 9)$ in case $(1.4)$. If we choose

$$
f(x, y)=a\left(a_{0}+3 x\right)^{3}+b\left(b_{0}+3 y\right)^{3}
$$


or

$$
f(x, y)=\frac{a\left(a_{0}+81 x\right)^{3}+b\left(b_{0}+81 y\right)^{3}}{27}
$$

in the two cases, our Theorem 2 produces a prime $p=f(x, y)$; by construction $p=2(\bmod 9)$, and the result follows.

Remark 3. The reader will note that neither Corollary 1, nor Corollary 2 can be deduced from our previous results on representation of primes by binary cubic forms obtained in [4]. In both cases the applications require congruence conditions on the primes to be represented.

\section{Some subsidiary estimates}

As in [4], let $\theta=\theta_{0} N\left(\omega_{1}\left(\omega_{1}, \omega_{2}\right)^{-1}\right)$; write $i(\theta)=(\mathfrak{o}: \mathbb{Z}[\theta])$. From now on we shall assume, without loss of generality, that h.c.f. $\left(\gamma_{1}, \gamma_{2}, d\right)=1$ and let $C=i(\theta) N\left(\omega_{1} \omega_{2}\right) d$. For $\vec{u} \in \mathbb{Z}^{2}$, let $\mathfrak{A}_{u}(\gamma):=(\gamma+u d) \mathfrak{d}^{-1}$, so that $f(\vec{x})=N_{k(\vec{x}) / \mathbb{Q}(\vec{x})} \mathfrak{A}_{x}(\gamma)$. Let

$$
\mathcal{A}=\left\{\mathfrak{A}_{a}(\gamma): \vec{a} \in I(X) \cap \mathbb{Z}^{2} \text {, h.c.f. }\left(\gamma_{1}+a_{1} d, \gamma_{2}+a_{2} d\right)=1\right\} .
$$

Let further

$$
\mathcal{R}=\left\{R: R \in \mathcal{I}, \mu(R)^{2}=1, \mu\left(\frac{N R}{N((R, C))}\right)^{2}=1\right\} .
$$

By [4, Lemma 2.2] (cf. also [3, Lemma 3.1]), if $\mathfrak{p}_{i}\left|\mathfrak{B}, \mathfrak{p}_{i}\right| p, \mathfrak{p}_{i} \in \mathcal{P}, i=1,2$, for some $\mathfrak{B}$ in $\mathcal{A}$ and some $p$ in $P$ and if $p$ is non-singular, that is if $p$ does not divide $i(\theta) N\left(\omega_{1} \omega_{2}\right)$, then $\mathfrak{p}_{1}=\mathfrak{p}_{2}$ and $N \mathfrak{p}_{1}=p$. Therefore, as it has been remarked in $[4$, p.261],

$$
\left(\left\{R: R \in \mathcal{I}, \mu(R)^{2}=1\right\} \cap \mathcal{A}\right) \subseteq \mathcal{R} .
$$

Let $A=\{N \mathfrak{a} \mid \mathfrak{a} \in \mathcal{A}\}$, then

$$
A=\left\{f(\vec{a}): \vec{a} \in I(X) \cap \mathbb{Z}^{2} \text {, h.c.f. }\left(\gamma_{1}+a_{1} d, \gamma_{2}+a_{2} d\right)=1\right\} .
$$

For $R \in \mathcal{R}$, let $r:=N R$ and

$$
\sigma(R, X ; \gamma, \vec{\beta})=\#\left\{\vec{u}: \vec{u} \in \mathbb{Z}^{2} \cap I(X, \vec{\beta}), R \mid \mathfrak{A}_{u}(\gamma)\right\}
$$

where $I(X, \vec{\beta}):=\{\vec{u}: \vec{u}+\vec{\beta} \in I(X)\}$, so that $I(X)=I(X, \overrightarrow{0})$. Further, let

$$
\mathfrak{m}(R, \gamma)=\left\{\vec{u}: \vec{u} \in \mathbb{Z}^{2}, \vec{u} \bmod r, R \mid \mathfrak{A}_{u}(\gamma)\right\},
$$




$$
m(R, \gamma)=\frac{1}{r} \# \mathfrak{m}(R, \gamma)
$$

and

$$
\sigma_{0}(R, a ; \gamma)=\sum_{\vec{u} \in \mathfrak{m}(R, \gamma)} e_{r}(\vec{a} \vec{u})
$$

so that

$$
m(R, \gamma)=\frac{\sigma_{0}(R, 0 ; \gamma)}{r} .
$$

As in [3, pp.28-29] and [4, p.261], it follows that

$$
\sigma(R, X ; \gamma, \vec{\beta})=r^{-2} \sum_{\substack{\vec{a} \bmod r, \vec{x} \in I(X, \vec{\beta}) \\ \vec{u} \in \mathfrak{m}(R, \gamma)}} e_{r}(\vec{a}(\vec{u}-\vec{x}))
$$

and therefore

$$
\sigma(R, X ; \gamma, \vec{\beta})=r^{-2} \sum_{\substack{\vec{a} \bmod r \\ \vec{x} \in I(X, \vec{\beta})}} \sigma_{0}(R, a ; \gamma) e_{r}(-\vec{a} \vec{x}) .
$$

Lemma 2.1. The sum $\sigma_{0}(R, a ; \gamma)$ is weakly multiplicative, that is

$$
\sigma_{0}(S T, a ; \gamma)=\sigma_{0}(S, a ; \gamma) \sigma_{0}(T, a ; \gamma)
$$

as soon as h.c.f. $\left(N_{k / \mathbb{Q}} S, N_{k / \mathbb{Q}} T\right)=1$.

Proof. Suppose that h.c.f. $\left(N_{k / \mathbb{Q}} S, N_{k / \mathbb{Q}} T\right)=1$. By the Chinese Remainder Theorem,

$$
\mathfrak{m}(S T, \gamma)=\mathfrak{m}(S, \gamma) \times \mathfrak{m}(T, \gamma)
$$

The assertion of the lemma follows from this relation and the definition of $\sigma_{0}(R, a ; \gamma)$.

By definition,

$$
\mathcal{A}_{R}=\left\{\mathfrak{A}_{a}: \vec{a} \in I(X) \cap \mathbb{Z}^{2} \text {, h.c.f. }\left(\gamma_{1}+a_{1} d, \gamma_{2}+a_{2} d\right)=1, R \mid \mathfrak{A}_{a}(\gamma)\right\} .
$$

For $R \in \mathcal{R}$, let

$$
\left[\mathcal{A}_{R}\right]:=\frac{\eta^{2} X^{2} m(R, \gamma)}{r} \sum_{(\delta, d)=1, \delta \in \mathbb{N}} \frac{\mu(\delta)}{\delta^{2}} \cdot \frac{N((R, \delta))}{m((R, \delta), \gamma)},
$$

and let

$$
\# \mathcal{A}_{R}=\left[\mathcal{A}_{R}\right]+\rho_{1}(R, X) \text {. }
$$


Lemma 2.2. For any positive integer $l$, there is a constant $c(l)$ such that

$$
\sum_{\substack{Q<N R \leq 2 Q \\ R \in \mathcal{R}}} \tau(R)^{l}\left|\rho_{1}(R, X)\right| \ll\left(Q+X Q^{1 / 2}+X^{3 / 2}\right)(\log Q X)^{c(l)} .
$$

Proof. Clearly,

$$
\# \mathcal{A}_{R}=\sum_{\delta \in \mathbb{N}} \mu(\delta) \# \mathcal{A}_{R}^{(\delta)}
$$

where

$$
\mathcal{A}_{R}^{(\delta)}:=\left\{\vec{a}: \vec{a} \in I(X) \cap \mathbb{Z}^{2}, \delta \mid \text { h.c.f. }\left(\gamma_{1}+a_{1} d, \gamma_{2}+a_{2} d\right), R \mid \mathfrak{A}_{a}(\gamma)\right\} .
$$

Since h.c.f. $\left(\gamma_{1}, \gamma_{2}, d\right)=1$, the summation in (2.5) may be restricted by the condition h.c.f. $(\delta, d)=1$.

Suppose that $R \in \mathcal{R}$ and let $R_{1}^{(\delta)}=\frac{R}{(R, \delta)}$ for $\delta \in \mathbb{N}$. It follows from Lemma 2.1 that

$$
m(R, \gamma)=m((R, \delta), \gamma) m\left(R_{1}^{(\delta)}, \gamma\right) .
$$

Assuming further that h.c.f. $(\delta, d)=1$ and $\mu(\delta)^{2}=1$, let $\beta^{(\delta)}$ be defined by the conditions:

$$
\delta \mid\left(\vec{\gamma}+\beta^{(\delta)} d\right), 0 \leq \beta_{1}^{(\delta)}, \beta_{2}^{(\delta)}<\delta .
$$

It can be easily seen that

$$
m\left(R_{1}^{(\delta)}, \gamma\right)=m\left(R_{1}^{(\delta)}, \frac{\gamma+\beta^{(\delta)} d}{\delta}\right)
$$

On substituting equations (2.6) and (2.7) in (2.3), one obtains

$$
\left[\mathcal{A}_{R}\right]:=\frac{\eta^{2} X^{2}}{r} \sum_{(\delta, d)=1, \delta \in \mathbb{N}} \frac{\mu(\delta)}{\delta^{2}} m\left(R_{1}^{(\delta)}, \frac{\gamma+\beta^{(\delta)} d}{\delta}\right) N((R, \delta)) .
$$

Moreover, equation (2.5) may be rewritten as follows

$$
\# \mathcal{A}_{R}=\sum_{\delta \in \mathbb{N},(\delta, d)=1} \mu(\delta) \# \mathcal{B}_{R}^{(\delta)}
$$

where

$$
\mathcal{B}_{R}^{(\delta)}:=\left\{\vec{b}: \vec{b} \in I\left(\frac{X}{\delta}, \frac{\beta^{(\delta)}}{\delta}\right) \cap \mathbb{Z}^{2}, R \mid \delta \mathfrak{A}_{b}\left(\frac{\gamma+\beta^{(\delta)} d}{\delta}\right)\right\} .
$$

In view of (2.1), this gives

$$
\# \mathcal{A}_{R}=\sum_{\delta \in \mathbb{N},(\delta, d)=1} \mu(\delta) \sigma\left(R_{1}^{(\delta)}, \frac{X}{\delta} ; \frac{\gamma+\beta^{(\delta)} d}{\delta}, \frac{\beta^{(\delta)}}{\delta}\right) .
$$


As in [3, p.29] and [4, p.261], it follows from (2.2) that

$$
\sigma(R, X ; \gamma, \vec{\beta})=\frac{\eta^{2} X^{2} m(R, \gamma)}{r}+\rho(R, X ; \gamma, \vec{\beta}),
$$

with

$$
\rho(R, X ; \gamma, \vec{\beta})=r^{-2} \sum_{\substack{\vec{a} \bmod r, \vec{a} \neq \overrightarrow{0} \\ \vec{x} \in I(X, \vec{\beta})}} \sigma_{0}(R, a ; \gamma) e_{r}(-\vec{a} \vec{x})+O\left(\frac{X m(R, \gamma)}{r}\right) .
$$

In view of (2.8) and (2.9), we conclude that (2.4) holds with

$$
\rho_{1}(R, X)=\sum_{\delta \in \mathbb{N},(\delta, d)=1} \mu(\delta) \rho\left(R_{1}^{(\delta)}, \frac{X}{\delta} ; \frac{\gamma+\beta^{(\delta)} d}{\delta}, \frac{\beta^{(\delta)}}{\delta}\right) .
$$

Since $m(S, \gamma)=1$ as soon as h.c.f. $(S, C)=1$, it follows from (2.6) that $m(R, \gamma)=m((R, C), \gamma)=O(1)$. Therefore

$$
\rho(R, X ; \gamma, \vec{\beta}))=\Sigma_{0}+O\left(\frac{X}{r}\right)
$$

with

$$
\Sigma_{0}=r^{-2} \sum_{\substack{\vec{a} \bmod r, \vec{a} \neq \overrightarrow{0} \\ \vec{x} \in I(X, \vec{\beta})}} \sigma_{0}(R, a ; \gamma) e_{r}(-\vec{a} \vec{x})
$$

Let

$$
\left.\Sigma_{1}=\sum_{\substack{Q<N R \leq 2 Q \\ R \in \mathcal{R}}} \tau(R)^{l} \mid \rho(R, X ; \gamma, \vec{\beta})\right) \mid .
$$

It follows from (2.12) that

$$
\Sigma_{1} \ll \Sigma_{11}+O\left(X(\log Q)^{c(l)}\right),
$$

where

$$
\Sigma_{11}:=\sum_{\substack{Q<N R \leq 2 Q \\ R \in \mathcal{R}}} \tau(R)^{A}\left|\Sigma_{0}\right|
$$

Clearly,

$$
\left|\Sigma_{0}\right| \leq r^{-2} \sum_{\vec{a} \bmod r, \vec{a} \neq 0}\left|\sigma_{0}(R, a ; \gamma)\right|\left|\sum_{\vec{x} \in I(X, \vec{\beta})} e_{r}(-\vec{a} \vec{x})\right|
$$


therefore it follows from Lemma 2.1 that

$$
\left|\Sigma_{0}\right| \ll \sum_{\vec{a} \in \Pi_{1}} f_{X}(R, a ; \gamma),
$$

where

$$
f_{X}(R, a ; \gamma):=\frac{\left|\sigma_{0}\left(R_{1}, a ; \gamma\right)\right|}{r^{2}} \min \left\{X, \frac{r}{\left|a_{1}\right|}\right\} \min \left\{X, \frac{r}{\left|a_{2}\right|}\right\}, \quad R_{1}:=\frac{R}{(R, C)},
$$

and

$$
\Pi_{1}=\left\{\vec{a}: \vec{a} \in \mathbb{Z}^{2}, \vec{a} \neq 0,\left|a_{1}\right|,\left|a_{2}\right| \leq r / 2\right\},
$$

cf. $[4,(3.6)]$. This gives

$$
\Sigma_{11} \ll \Sigma_{12}+\Sigma_{13}
$$

with

$$
\Sigma_{1 j}=\sum_{\substack{Q<r \leq 2 Q \\ R \in \mathcal{R}, \vec{a} \in \Pi_{j}}} \tau(R)^{l} f_{X}(R, a ; \gamma), j=2,3,
$$

where

$$
\Pi_{2}=\left\{\vec{a}: \vec{a} \in \Pi_{1}, a_{1} a_{2}=0\right\}, \Pi_{3}=\left\{\vec{a}: \vec{a} \in \Pi_{1}, a_{1} a_{2} \neq 0\right\} .
$$

As in [3, p.30] and [4, p.262], it follows that

$$
\Sigma_{12} \ll X \sum_{0<a \leq Q} \frac{1}{a} \sum_{\substack{Q<r \leq 2 Q \\ R_{1} \mid a}} \tau(R)^{l} \ll X \sum_{0<a \leq Q} \frac{\tau(a)^{c_{1}(l)}}{a} ;
$$

thus

$$
\Sigma_{12} \ll X(\log Q)^{c(l)} .
$$

Clearly,

$$
\Sigma_{13} \leq \sum_{\substack{Q<r \leq 2 Q \\ R \in \mathcal{R}, \vec{a} \in \Pi_{3}}} \tau(R)^{l} \frac{\left|\sigma_{0}\left(R_{1}, a ; \gamma\right)\right|}{\left|a_{1} a_{2}\right|} .
$$

Suppose that $\mathfrak{p} \in \mathcal{R}, N \mathfrak{p}=p, p \in P$, and $C \neq 0 \bmod p$, then

$$
\left|\sigma_{0}(\mathfrak{p}, a ; \gamma)\right|=\left|\sum_{\vec{u} \bmod p, \mathfrak{p} \mid \gamma+u d} e_{p}(\vec{u} \vec{a})\right|=\left|\sum_{\vec{v} \bmod p, \mathfrak{p} \mid v} e_{p}(\vec{v} \vec{a})\right| .
$$


Therefore the scaling argument in $[3, \S 5 ; 4, \S 3]$ shows that

$$
\sigma_{0}\left(R_{1}, \vec{a} ; \gamma\right) \neq 0 \Rightarrow a_{2} \omega_{1}=a_{1} \omega_{2} \quad \bmod R_{1}
$$

cf. $[4,(3.7)]$. This gives

$$
\Sigma_{13} \leq \sum_{\substack{Q<r \leq 2 Q \\ R \in \mathcal{R}, \vec{a} \in \Omega}} \tau(R)^{l} \frac{\left|\sigma_{0}\left(R_{1}, a ; \gamma\right)\right|}{\left|a_{1} a_{2}\right|},
$$

where

$$
\Omega=\left\{\vec{a}: \vec{a} \in \mathbb{Z}^{2}, 1 \leq\left|a_{1}\right|,\left|a_{2}\right| \leq Q, a_{2} \omega_{1}=a_{1} \omega_{2} \quad \bmod R_{1}\right\}
$$

since

$$
\left|\sigma_{0}\left(R_{1}, a ; \gamma\right)\right| \ll m\left(R_{1}, \gamma\right) r \ll Q
$$

it follows that

$$
\Sigma_{13} \leq Q \sum_{\substack{Q<r \leq 2 Q \\ R \in \mathcal{R}, \vec{a} \in \Omega}} \frac{\tau(R)^{l}}{\left|a_{1} a_{2}\right|}
$$

Thus

$$
\Sigma_{13} \ll Q(\log Q)^{c(l)},
$$

as in [3, p.30] and [4, pp.262-263]. Relations (2.13-16) give

$$
\Sigma_{1} \ll(X+Q)(\log Q)^{c(l)} .
$$

Let

$$
\Sigma_{2}=\sum_{\substack{Q<N R \leq 2 Q \\ R \in \mathcal{R}}} \tau(R)^{l}\left|\rho_{1}(R, X)\right|
$$

and

$$
g_{X}(R, \delta ; \gamma):=\left|\rho\left(R_{1}^{(\delta)}, \frac{X}{\delta} ; \frac{\gamma+\beta^{(\delta)} d}{\delta}, \frac{\beta^{(\delta)}}{\delta}\right)\right| .
$$

In view of $(2.11)$,

$$
\Sigma_{2} \leq \sum_{\substack{Q<r \leq 2 Q \\ R \in \mathcal{R}, \delta \in \Omega_{1}}} \tau(R)^{l} g_{X}(R, \delta ; \gamma)
$$


where

$$
\Omega_{1}:=\{\delta: \delta \in \mathbb{N} \text {, h.c.f. }(\delta, d)=1\} \text {. }
$$

For $\Delta>0$, let

$$
\Omega_{1}^{+}:=\left\{\delta: \delta \in \Omega_{1}, \delta>\Delta\right\}, \Omega_{1}^{-}:=\Omega_{1} \backslash \Omega_{1}^{+} .
$$

It follows from (2.18) that

$$
\Sigma_{2} \leq \Sigma_{21}+\Sigma_{22}
$$

where

$$
\Sigma_{21}=\sum_{\substack{Q<r \leq 2 Q \\ R \in \mathcal{R}, \delta \in \Omega_{1}^{+}}} \tau(R)^{l} g_{X}(R, \delta ; \gamma)
$$

and

$$
\Sigma_{22}=\sum_{\substack{Q<r \leq 2 Q \\ R \in \mathcal{R}, \delta \in \Omega_{1}^{-}}} \tau(R)^{l} g_{X}(R, \delta ; \gamma) .
$$

Further, it follows from (2.10) that

$$
\Sigma_{21} \leq \Sigma_{23}+\Sigma_{24}
$$

with

$$
\Sigma_{23}=\sum_{\substack{Q<r \leq 2 Q \\ R \in \mathcal{R}, \delta \in \Omega_{1}^{+}}} \tau(R)^{l} \sigma\left(R_{1}^{(\delta)}, \frac{X}{\delta} ; \frac{\gamma+\beta^{(\delta)} d}{\delta}, \frac{\beta^{(\delta)}}{\delta}\right)
$$

and

$$
\Sigma_{24}=\sum_{\substack{Q<r \leq 2 Q \\ R \in \mathcal{R}, \delta \in \Omega_{1}^{+}}} \tau(R)^{l} \frac{\eta^{2} X^{2}}{\delta^{2} N R_{1}^{(\delta)}} m\left(R_{1}^{(\delta)}, \frac{\gamma+\beta^{(\delta)} d}{\delta}\right) .
$$

Since $m(R, \gamma)=O(1)$, we have

$$
\Sigma_{24} \ll \eta^{2} X^{2} \sum_{\substack{Q<r \leq 2 Q \\ R \in \mathcal{R}, \delta>\Delta}} \frac{\tau(R)^{l} N((R, \delta))}{\delta^{2} N R} .
$$

The argument proceeds as in [4, p.264]. One first obtains the following estimate

$$
\Sigma_{24} \ll \eta^{2} X^{2} \Delta^{-1}(\log Q)^{c(l)}
$$


Then we let

$$
R_{0}:=\text { h.c.f. }(R, C), R=R_{1} R_{0}, \mathfrak{A}_{1}=\text { h.c.f. }\left(R_{1}, \delta\right), R_{1}=\mathfrak{A}_{1} \mathfrak{A}_{2}
$$

and conclude that

$$
\Sigma_{23} \ll \sum_{\delta \in \Omega_{1}^{+}, N \mathfrak{A}_{1} \mid \delta} \tau\left(\mathfrak{A}_{1}\right)^{l} \Sigma_{25}\left(\mathfrak{A}_{1}\right)
$$

where

$$
\Sigma_{25}\left(\mathfrak{A}_{1}\right)=\sum_{Q<N\left(\mathfrak{A}_{1} \mathfrak{A}_{2} R_{0}\right) \leq 2 Q} \tau\left(\mathfrak{A}_{2}\right)^{l} \sigma\left(\mathfrak{A}_{2}, \frac{X}{\delta} ; \frac{\gamma+\beta^{(\delta)} d}{\delta}, \frac{\beta^{(\vec{\delta})}}{\delta}\right) .
$$

Moreover, the definition (2.1) of $\sigma(R, X ; \gamma, \vec{\beta})$ shows that, as in [4, p.264], one may apply relation $[4,(3.8)]$ to obtain the following upper bound:

$$
\Sigma_{25}\left(\mathfrak{A}_{1}\right) \ll X^{2} \delta^{-2}(\log X)^{c_{1}(l)} .
$$

Therefore (2.22) gives

$$
\Sigma_{23} \ll \sum_{\delta>\Delta, N \mathfrak{A}_{1} \mid \delta} \tau\left(\mathfrak{A}_{1}\right)^{l} X^{2} \delta^{-2}(\log X)^{c_{1}(l)}
$$

thus

$$
\Sigma_{23} \ll X^{2} \Delta^{-1}(\log X)^{c(l)} .
$$

On the other hand (cf. [4, p.264]),

$$
\Sigma_{22} \ll \sum_{\delta \in \Omega_{1}^{-}, N R_{1} \mid \delta} \tau(R)^{l} \Sigma_{26}(R),
$$

with

$$
\Sigma_{26}(R)=\sum_{\substack{Q<N(R S) \leq 2 Q, R S \in \mathcal{R}}} \tau(S)^{l}\left|\rho\left(S, \frac{X}{\delta} ; \frac{\gamma+\beta^{(\delta)} d}{\delta}, \frac{\beta^{(\delta)}}{\delta}\right)\right| .
$$

It follows from the relations (2.24) and (2.17) that

$$
\Sigma_{22} \ll \sum_{\delta \leq \Delta, N R_{1} \mid \delta} \tau(R)^{l}\left(\frac{X}{\delta}+\frac{Q}{N R}\right)(\log Q)^{c_{2}(l)} ;
$$

where in the above notation $R=R_{1} R_{0}$. As in $[3, \S 5]$, this gives

$$
\Sigma_{22} \ll \Delta(X+Q)(\log Q)^{c(l)}
$$


for $\Delta \ll X$. Put

$$
\Delta=1+\min \left\{X^{1 / 2}, X Q^{-1 / 2}\right\}
$$

the asserted estimate

$$
\Sigma_{2} \ll\left(Q+X Q^{1 / 2}+X^{3 / 2}\right)(\log Q X)^{c(l)}
$$

follows from relations (2.19)-(2.21), (2.23), and (2.25). This concludes the proof of Lemma 2.2.

Let now

$$
\left[A_{q}\right]=\mu(q) \sum_{\substack{R|q, q| N R \\ R \in \mathcal{R}}} \mu(R)\left[\mathcal{A}_{R}\right]
$$

and

$$
\rho_{2}(q, X)=\mu(q) \sum_{\substack{R|q, q| N R \\ R \in \mathcal{R}}} \mu(R) \rho_{1}(R, X)
$$

for $q \in \mathbb{N}$.

Lemma 2.3 If $\mu(q)^{2}=1$, then

$$
\# A_{q}=\left[A_{q}\right]+\rho_{2}(q, X) ;
$$

moreover, for any positive integer $l$, there is a constant $c(l)$ such that

$$
\sum_{Q<q \leq 2 Q} \tau(q)^{A} \mu(q)^{2}\left|\rho_{2}(q, X)\right| \ll\left(Q+X Q^{1 / 2}+X^{3 / 2}\right)(\log Q X)^{c(l)} .
$$

Proof. As in [3, pp.32-33] and [4, p.265], assuming $q$ be square-free, we deduce from the identity

$$
\mu(q) \sum_{\substack{R|\mathfrak{A}, R| q \\
q \mid N R}} \mu(R)=\left\{\begin{array}{lc}
1, & q \mid N \mathfrak{A}, \\
0, & \text { otherwise }
\end{array}\right.
$$

that

$$
\# A_{q}=\mu(q) \sum_{\substack{R|q, q| N R \\ R \in \mathcal{R}}} \mu(R) \# \mathcal{A}_{R} .
$$

Therefore equation (2.27) follows from (2.4). The estimate (2.28) follows from Lemma 2.2 in exactly the same way as the corresponding estimate in [4, Lemma 3.3]. 
Let

$$
\zeta_{d}(s)=\zeta(s) \prod_{p \in P, p \mid d}\left(1-\frac{1}{p^{s}}\right)
$$

for $s \in \mathbb{C}$. As in [3, p.5] and [4, p.266], let $\nu_{p}:=\{\mathfrak{p}: \mathfrak{p} \in \mathcal{P}, N \mathfrak{p}=p\}$ stand for the number of prime ideals of the first degree in $k$ lying above $p$.

Lemma 2.4 There are a multiplicative function $\Gamma(q)$ and a positive real number $c_{0}$ satisfying the following conditions:

(i) $0 \leq \Gamma(p) \leq 1$ for $p \in P$;

(ii) $1-\Gamma(p) \geq c_{0}$ as soon as $p>3, p \in P$;

(iii) if $\mu(q)^{2}=1$, then

$$
\left[A_{q}\right]=\frac{\eta^{2} X^{2}}{\zeta_{d}(2)} \Gamma(q)
$$

(iv) if $C \neq 0 \bmod p$, then

$$
\Gamma(p)=\frac{\nu_{p}}{p+1}
$$

Moreover, $\varepsilon(f) \in\{1,2,3,6\}$ and

$$
1-\Gamma(p)=0 \Leftrightarrow p \mid \varepsilon(f)
$$

for $p=2,3$.

Proof. Let $R \in \mathcal{R}$. Since $m(R, \gamma)=m\left(R_{0}, \gamma\right)$, it follows from the definition (2.3) that

$$
\left[\mathcal{A}_{R}\right]=\frac{\eta^{2} X^{2}}{\zeta_{d}(2) N R} \tilde{m}(R, \gamma)
$$

with

$$
\tilde{m}(R, \gamma)=m\left(R_{0}, \gamma\right) \prod_{p \in P_{0}(R, d)}\left(1-\frac{1}{p^{2}}\right)^{-1}\left(1-\frac{N((R, p))}{p^{2} m((R, p), \gamma)}\right),
$$

where

$$
P_{0}(R, d)=\{p: p \in P, p \mid N R, d \neq 0 \bmod p\}
$$

On letting

$$
\Gamma(q)=\mu(q) \sum_{\substack{R|q, q| N R \\ R \in \mathcal{R}}} \frac{\mu(R)}{N R} \tilde{m}(R, \gamma),
$$

one deduces equation (2.29) from relations (2.26) and (2.30). Clearly,

$$
\Gamma(q)=\prod_{p \in P, p \mid q} \Gamma(p)
$$


and

$$
\Gamma(p)=-\sum_{\substack{R|p, p| N R \\ R \in \mathcal{R}}} \frac{\mu(R)}{N R} \tilde{m}(R, \gamma)
$$

for $p \in P$. Let

$$
b(R):=p^{2} m(R, \gamma) N R^{-1}
$$

and note that

$$
b(R)=\#\left\{\vec{u}: \vec{u} \in \mathbb{Z}^{2}, 0 \leq u_{1}, u_{2}<p, R \mid \mathfrak{A}_{u}(\gamma)\right\},
$$

if $R \mid p, p \in P$; in particular, $b(1)=p^{2}$. One concludes from (2.34) that

$$
\sum_{R \mid p} \mu(R) b(R)=\#\left\{\vec{u}: \vec{u} \in \mathbb{Z}^{2}, 0 \leq u_{1}, u_{2}<p \text {, h.c.f. }\left(\mathfrak{A}_{u}(\gamma), p\right)=1\right\} \text {. }
$$

Suppose first $C \neq 0 \bmod p$. Then

$$
\Gamma(p)=\frac{\nu_{p}}{p+1}
$$

and, consequently, $0 \leq \Gamma(p) \leq 3 / 4$ for $p \in P$ since $\nu_{2} \leq 2$ when 2 does not divide $i(\theta)$ (cf. [5]).

Suppose now that $p \mid C$ but $d \neq 0 \bmod p$; then it follows from (2.31-33) that

$$
\Gamma(p)=\frac{1}{1-p^{2}} \sum_{\substack{R \mid p, R \neq 1 \\ R \in \mathcal{R}}} \mu(R)(b(R)-1) .
$$

Since $b(1)=p^{2}$, the last equation may be rewritten as follows:

$$
\Gamma(p)=\frac{1}{1-p^{2}} \sum_{R \mid p} \mu(R) b(R)+1 .
$$

Moreover, since $d \neq 0 \bmod p$, it follows from (2.35) that

$$
\sum_{R \mid p} \mu(R) b(R)=\#\left\{\vec{u}: \vec{u} \in \mathbb{Z}^{2}, 0 \leq u_{1}, u_{2}<p \text {, h.c.f. }\left(\tilde{\mathfrak{A}}_{u}(\gamma), p\right)=1\right\},
$$

where

$$
\left.\tilde{\mathfrak{A}}_{u}(\gamma):=\left(u_{1} \omega_{1}+u_{2} \omega_{2}\right) \text { (h.c.f. }\left(\omega_{1}, \omega_{2}\right)\right)^{-1} .
$$

Hence $\Gamma(p)$ depends neither on $d$, nor on $\gamma$, if $p$ does not divide $d$; therefore all the assertions of the lemma follow from [4, Lemma 3.4] in this case. 
Let now $p \mid d$, then it follows from (2.31) that $\tilde{m}(R, \gamma)=m\left(R_{0}, \gamma\right)$ for $R \mid p$; therefore equation (2.32) may be rewritten as follows:

$$
\Gamma(p)=-\sum_{\substack{R|p, p| N R \\ R \in \mathcal{R}}} \mu(R) \frac{m(R, \gamma)}{N R},
$$

or

$$
1-\Gamma(p)=\frac{1}{p^{2}} \sum_{R \mid p} \mu(R) b(R) .
$$

Hence $0 \leq 1-\Gamma(p) \leq 1$, in view of $(2.35)$; this proves (i). Let

$$
d=p^{\mu_{0}} d_{0}, p=\mathfrak{p}^{e} \mathfrak{q}, \gamma=\mathfrak{p}^{\mu_{1}} \mathfrak{b}_{1} \text {, h.c.f. }\left(\omega_{1}, \omega_{2}\right)=\mathfrak{p}^{\mu_{2}} \mathfrak{b}_{2}
$$

with $\mathfrak{p} \in \mathcal{P}, \quad d_{0} \mathfrak{b}_{1} \mathfrak{b}_{2} \mathfrak{q} \neq 0 \bmod \mathfrak{p}$. Then h.c.f. $\left(\mathfrak{A}_{u}(\gamma), \mathfrak{p}\right)=1$ unless $\mu_{1} \geq$ $e \mu_{0}+\mu_{2} ;$ moreover, if $\mu_{1} \geq e \mu_{0}+\mu_{2}$, then

$$
\#\left\{\vec{u}: \vec{u} \in \mathbb{Z}^{2}, 0 \leq u_{1}, u_{2}<p, \mathfrak{p} \mid \mathfrak{A}_{u}(\gamma)\right\} \leq p .
$$

Hence

$$
\#\left\{\vec{u}: \vec{u} \in \mathbb{Z}^{2}, 0 \leq u_{1}, u_{2}<p \text {, h.c.f. }\left(\mathfrak{A}_{u}(\gamma), p\right) \neq 1\right\} \leq 3 p ;
$$

therefore it follows from (2.35) and (2.36) that

$$
1-\Gamma(p) \geq 1-\frac{3}{p}>0
$$

for $p>3$; this proves (ii). Moreover, it is clear also that $\Gamma(p)=1$ if and only if h.c.f. $\left(\mathfrak{A}_{u}(\gamma), p\right) \neq 1$ for every $\vec{u}$ in $\mathbb{Z}^{2}$. Thus $\varepsilon(f)$ is divisible by no prime $p>3$. An analogous argument shows that $\varepsilon(f)$ is square-free. This completes the proof of Lemma 2.4.

\section{The sieve bounds}

As in $[4,(3.17)]$, let

$$
\sigma(f)=\prod_{p \in P}\left(1+\frac{1}{p}\right)(1-\Gamma(p)) ;
$$

in view of Lemma 2.4(iv), the product $\sigma(f)$ converges (cf. [4, Lemma 3.4]). Let

$$
\sigma_{1}(f)=\sigma(f) \prod_{p \in P, p \mid d}\left(1-\frac{1}{p^{2}}\right)^{-1},
$$


$h_{f_{0}}=f_{0}(1,1), h_{f}=d^{3} h_{f_{0}}$, and $\kappa=\sigma_{1}(f) \eta\left(h_{f} X\right)^{-1}$. In what follows, it will be assumed that $\sigma(f) \neq 0$ (and therefore $\kappa \neq 0$ ).

Let

$$
\mathcal{B}=\left\{\mathfrak{A}: \mathfrak{A} \in \mathcal{I}, h_{f} X^{3}<N \mathfrak{A} \leq h_{f} X^{3}(1+\eta)\right\},
$$

and $B=\{N \mathfrak{A}: \mathfrak{A} \in \mathcal{B}\}$. Write, for brevity, $\tau:=(\log \log X)^{-1 / 6}$. The asymptotic formula (1.2) of Theorem 2 is equivalent to the following estimate to be proved along the lines of $[3,4]$ :

$$
\pi(\mathcal{A})=\kappa \pi(\mathcal{B})+O\left(\frac{\eta^{2} X^{2}}{\log X} \tau\right)
$$

Lemmata 3.5, 3.6, and 4.1 in [4] can be taken over verbatim. Let us prove the analogue of [4, Lemma 4.2] (cf. also [3, Lemma 3.5]). We adopt the sieve notation introduced in $[4, \S 4]$.

Lemma 3.1 We have

$$
\sum_{0 \leq n \leq n_{0}}\left|T^{(n)}(\mathcal{A})-\kappa T^{(n)}(\mathcal{B})\right| \ll \tau \frac{\eta^{2} X^{2}}{\log X} .
$$

Proof. Without loss of generality, it may be assumed that

$$
p \geq X^{\tau} \Rightarrow C \neq 0 \bmod p .
$$

Let

$$
j(q)=\mu(q) q \sum_{R|q, q| N R} \frac{\mu(R)}{N R}=q \prod_{p \in P, p \mid q}\left(1-\prod_{\mathfrak{p} \in \mathcal{P}, \mathfrak{p} \mid p}\left(1-\frac{1}{N \mathfrak{p}}\right)\right),
$$

cf. [4, Lemma 3.6]. Equation [4, (4.9)] gives

$$
\begin{gathered}
T^{(n)}(\mathcal{B})=\phi(k) h_{f} \eta X^{3} \prod_{p<X^{\tau}}\left(1-\frac{j(p)}{p}\right) \Sigma_{5}^{(n)}\left\{1+O\left(\exp \left(-\tau^{-1}\right)\right)\right\} \\
+O\left(X^{3-\tau / 3}\right)
\end{gathered}
$$

where $\phi(k)$ stands for the residue of the zeta-function $\zeta_{k}(s)$ of $k$ at $s=1$ (cf. $[4, \mathrm{p} .258])$ and

$$
\Sigma_{5}^{(n)}=\sum_{\substack{X^{\tau} \leq p_{n}<\ldots<p_{1}<X^{1-\tau} \\ p_{1} \ldots p_{n}<X^{1+\tau}}} \frac{j\left(p_{1} \ldots p_{n}\right)}{p_{1} \ldots p_{n}}
$$

In view of (3.3), it follows from [4, Lemma 2.2] that

$$
S\left(\mathcal{A}_{\lambda(\overrightarrow{\mathfrak{p}})}, X^{\tau}\right)=S\left(A_{p_{1} \ldots p_{n}}, X^{\tau}\right)
$$


for $\overrightarrow{\mathfrak{p}} \in J_{n}(\mathcal{A})$ with $N \mathfrak{p}_{i}=p_{i}, p_{i} \in P, 1 \leq i \leq n$, and $\lambda(\overrightarrow{\mathfrak{p}})=\prod_{i=1}^{n} \mathfrak{p}_{i}$. Therefore

$$
T^{(n)}(\mathcal{A})=\sum_{\substack{X^{\tau} \leq p_{n}<\ldots<p_{1}<X^{1-\tau} \\ p_{1} \ldots p_{n}<X^{1+\tau}}} S\left(A_{p_{1} \ldots p_{n}}, X^{\tau}\right), T^{(0)}(\mathcal{A})=S\left(\mathcal{A}, X^{\tau}\right) .
$$

By the 'Fundamental Lemma', [1, Theorem 7.1], with ' $\omega(p)$ ' $=p \Gamma(p)$, ' $X$ ' $=$ $\frac{\eta^{2} X^{2}}{\zeta_{d}(2)},{ }^{\prime} \xi '=X^{1 / 6}$, and ' $z$ ' $=X^{\tau}$, it follows from Lemmata 2.3 and 2.4 that

$$
S\left(A_{q}, X^{\tau}\right)=M(q)\left\{1+O\left(\exp \left(-\tau^{-1}\right)\right)\right\}+O(E(q))
$$

where

$$
M(q)=\Gamma(q) \prod_{p<X^{\tau}}(1-\Gamma(p)) \frac{\eta^{2} X^{2}}{\zeta_{d}(2)}
$$

and

$$
E(q)=\sum_{\substack{\delta<X^{1 / 3} \\ p \mid \delta \Rightarrow p<X^{\tau}}} \mu(\delta)^{2} \tau(\delta)^{2}\left|\rho_{2}(q \delta, X)\right|
$$

This gives

$$
\begin{gathered}
T^{(n)}(\mathcal{A})=\frac{\eta^{2} X^{2}}{\zeta_{d}(2)} \prod_{p<X^{\tau}}(1-\Gamma(p)) \Sigma_{4}^{(n)}\left(1+O\left(\exp \left(-\tau^{-1}\right)\right)\right) \\
+\rho_{3}(X)
\end{gathered}
$$

with

$$
\Sigma_{4}^{(n)}=\sum_{\substack{X^{\tau} \leq p_{n}<\ldots<p_{1}<X^{1-\tau} \\ p_{1} \ldots p_{n}<X^{1+\tau}}} \Gamma\left(p_{1} \ldots p_{n}\right)
$$

and

$$
\rho_{3}(X)=\sum_{q<X^{4 / 3+\tau}} \mu(q)^{2} \tau(q)^{2}\left|\rho_{2}(q, X)\right| .
$$

It follows from $(2.28)$ that $\rho_{3}(X) \ll X^{7 / 4}(\log X)^{c}$. Moreover, as in [3, p.67], it follows from (2.28) that

$$
\prod_{p<X^{\tau}}(1-\Gamma(p))=\sigma(f) \zeta(2) \prod_{p<X^{\tau}}\left(1-\frac{1}{p}\right)\left(1+O\left(\left(\log X^{\tau}\right)^{-2}\right)\right),
$$


cf. $[3,(6.7)]$, and

$$
\prod_{p<X^{\tau}}\left(1-\frac{j(p)}{p}\right)=\phi(k)^{-1} \prod_{p<X^{\tau}}\left(1-\frac{1}{p}\right)\left(1+O\left(\left(\log X^{\tau}\right)^{-2}\right)\right),
$$

cf. $[3,(6.9)]$. Combining these estimates, one deduces from (3.6) and (3.4) that

$$
\begin{gathered}
T^{(n)}(\mathcal{A})= \\
\frac{\eta^{2} X^{2} \sigma(f) \zeta(2)}{\zeta_{d}(2)} \prod_{p<X^{\tau}}\left(1-\frac{1}{p}\right) \Sigma_{4}^{(n)}\left(1+O\left(\exp \left(-\tau^{-1}\right)\right)\right)\left(1+O\left(\left(\log X^{\tau}\right)^{-2}\right)\right) \\
+O\left(X^{7 / 4}(\log X)^{c}\right)
\end{gathered}
$$

and

$$
\begin{gathered}
T^{(n)}(\mathcal{B})=h_{f} \eta X^{3} \prod_{p<X^{\tau}}\left(1-\frac{1}{p}\right) \Sigma_{5}^{(n)}\left(1+O\left(\exp \left(-\tau^{-1}\right)\right)\right)\left(1+O\left(\left(\log X^{\tau}\right)^{-2}\right)\right) \\
+O\left(X^{3-\tau / 3}\right)
\end{gathered}
$$

The assertion of the lemma can be deduced from the relations (3.7) and (3.8) in exactly the same way as the analogous estimate in $[3, \S 6]$.

Neither the formulation, nor the proof of the analogues of [3, Lemmata 3.6 and 3.7] (cf. also [4, Lemmata 4.4 and 5.1]) need be changed. For the reader's convenience, we reproduce the assertions of these Lemmata to be deduced as in $[3, \S 7]$ from a variant of Selberg's upper bound sieve, see $[1$, Theorem 4.1].

Lemma 3.2 We have

$$
S_{j}(\mathcal{A})+\kappa S_{j}(\mathcal{B}) \ll \tau \frac{\eta^{2} X^{2}}{\log X}
$$

for $j=3,5,6$ or 7 .

Lemma 3.3 We have

$$
\begin{gathered}
\sum_{n \geq 3}\left|U^{(n)}(\mathcal{D})-\hat{U}^{(n)}(\mathcal{D})\right| \ll \frac{\eta^{2} X^{2}}{\log X} \xi \tau^{-4}, \\
\left|U_{1}^{(n)}(\mathcal{D})-\hat{U}_{1}^{(n)}(\mathcal{D})\right| \ll \frac{\eta^{2} X^{2}}{\log X} \xi \tau^{-3}
\end{gathered}
$$

for $n=1$ and 2 ,

$$
\left|S_{4}(\mathcal{D})-\hat{S}_{4}(\mathcal{D})\right| \ll \frac{\eta^{2} X^{2}}{\log X} \xi \tau^{-3}
$$


and

$$
\left|U_{2}^{(1)}(\mathcal{D})-\hat{U}_{2}^{(1)}(\mathcal{D})\right| \ll \frac{\eta^{2} X^{2}}{\log X} \xi \tau^{-4},
$$

where $\mathcal{D}$ stands for either of the sequences $\mathcal{A}, \mathcal{B}$.

Remark 4. Lemmata 3.1-3 may be regarded as formal corollaries of Lemmata 2.3 and 2.4, defining the 'level of distribution' of the sequence $\mathcal{A}$. We expect analogous assertions to be provable for other sequences of ideals in $k$ with the same level of distributon, in particular, for the sequence arising if a general cubic polynomial in $\mathbb{Z}\left[x_{1}, x_{2}\right]$ completely decomposable over $\mathbb{C}$ is studied. As it has been stressed in $[3, \S 2]$, the real novelty of the method developed in [3] is the 'Type II' estimates to be treated in the next section.

\section{The Type II estimates}

The strategy of the Type II estimates was outlined in [3, pp.9-10] and explained in detail in [3, pp.14-21]. Let us retain the notations introduced in [4, pp.271-273]; in particular, each of the relevant sums $U(\mathcal{A}), V(\mathcal{A})$ is decomposed as follows (cf. [4, p.273]):

$$
U(\mathcal{A})=U_{e}(\mathcal{A})+U_{g}(\mathcal{A}), V(\mathcal{A})=V_{e}(\mathcal{A})+V_{g}(\mathcal{A}) .
$$

We start with following analogue of [3, Lemma 3.9; 4, Lemma 5.2].

Lemma 4.1 In notation of $[4, \S 5]$, suppose that $\vec{m} \in \iota(n)$ and $\nu \in \iota_{1}(n)$. Then there is a constant $c$, depending at most on $f$ and such that

$$
U_{e}(\mathcal{A})-\kappa U(\mathcal{B}) \ll M^{-1} \eta^{5 / 2} X^{2}(\log X)^{c},
$$

where $M=\prod_{i=1}^{n+1} m_{i}$. Moreover,

$$
V_{e}(\mathcal{A})-\kappa V(\mathcal{B})=O\left(\eta^{5 / 2} X^{2}(\log X)^{c}\right),
$$

and

$$
\sum_{n \geq 3}\left|\hat{U}_{e}^{(n)}(\mathcal{A})-\kappa \hat{U}^{(n)}(\mathcal{B})\right|=O\left(\eta^{5 / 2} X^{2}(\log X)^{c}\right) .
$$

Proof. For $n \geq 0$, let $\vec{m} \in \iota(n)$ and $\nu \in \iota_{1}(n)$. As in [3, $\left.\S 10\right]$ and [4, p.274], one can deduce from $[3,(8.3)]$ and $[4,(3.8)]$ in the case $n>0$ and from (2.30) in conjunction with Lemma 2.2 when $n=0$ that, for a suitable constant $c_{1}$,

$$
U_{e}(\mathcal{A})=\sum_{\mathfrak{A} \mathfrak{D} \in \mathcal{R}, N \mathfrak{D}<L} a(\mathfrak{A}, \mathfrak{D}) \# \mathcal{A}_{\mathfrak{A D}}+O\left(\frac{\eta^{5 / 2} X^{2}}{M}(\log X)^{c_{1}}\right),
$$


where $L=X^{\tau / 2}$ and

$$
a(\mathfrak{A}, \mathfrak{D})=b_{\mathfrak{A}} \frac{w^{\prime}\left(h_{f} X^{3} / N(\mathfrak{A})\right)}{M(\xi \log X)^{n+1}} \mu(\mathfrak{D}) \log \frac{L}{N \mathfrak{D}} .
$$

It follows from the definitions that

$$
w^{\prime}(t) \neq 0 \Rightarrow X^{1+\tau} \leq t<X^{3 / 2-\tau}
$$

since

$$
(1+\tau) \xi^{-1} \leq \sum_{i=1}^{n+1} m_{i} \leq\left(\frac{3}{2}-\tau\right) \xi^{-1}-(n+1)
$$

when $\vec{m} \in \iota(n) \nu \in \iota_{1}(n)$, cf. $[4, \S 5]$. Therefore it may be assumed that the summation in (4.1) is subject to the additional condition

$$
N(\mathfrak{A D}) \ll X^{2-\tau / 2} .
$$

It follows from (2.4) and (2.30) that

$$
\# \mathcal{A}_{\mathfrak{A D}}=\frac{\eta^{2} X^{2}}{\zeta_{d}(2) N(\mathfrak{A D})} \tilde{m}(\mathfrak{A D}, \gamma)+\rho_{1}(\mathfrak{A D}, X) .
$$

As in [3, p.62] and [4, p.274], one deduces from (4.1)-(4.3) and Lemma 2.2 that, for some $c_{2}$,

$$
\begin{aligned}
U_{e}(\mathcal{A}) & =\frac{\eta^{2} X^{2}}{\zeta_{d}(2) M(\xi \log X)^{n+1}} \sum_{\mathfrak{A} \in \mathcal{R}} b_{\mathfrak{A}} \frac{w^{\prime}\left(h_{f} X^{3} / N \mathfrak{A}\right)}{N \mathfrak{A}} \tilde{m}(\mathfrak{A}, \gamma) \Sigma_{6} \\
& +O\left(\frac{\eta^{5 / 2} X^{2}}{M}(\log X)^{c_{2}}\right)
\end{aligned}
$$

with

$$
\Sigma_{6}=\sum_{\mathfrak{D} \in \mathcal{R}, N \mathfrak{D}<L} \frac{\mu(\mathfrak{D})}{N \mathfrak{D}} \tilde{m}(\mathfrak{D}, \gamma) \log \frac{L}{N \mathfrak{D}} .
$$

As in $[3$, p.62-63] and $[4$, p.274], it follows now from Perron's formula that

$$
\Sigma_{6}=\prod_{p \in P}\left(1-\frac{1}{p}\right)^{-1}(1-\Gamma(p))+O\left(\exp \left(-c_{3}(\log L)^{1 / 2}\right)\right),
$$

with $c_{3}>0$. In view of $(3.1)$, one concludes that

$$
U_{e}(\mathcal{A})=\frac{\sigma(f) \eta^{2} X^{2} \zeta(2)}{\zeta_{d}(2)} \Sigma_{7}\left(1+O\left(\exp \left(-c(\log L)^{1 / 2}\right)\right)\right)
$$




$$
+O\left(M^{-1} \eta^{5 / 2} X^{2}(\log X)^{c}\right)
$$

where

$$
\Sigma_{7}=\sum_{\mathfrak{A} \in \mathcal{R}} b_{\mathfrak{A}} \frac{w^{\prime}\left(h_{f} X^{3} / N(\mathfrak{A})\right)}{M(\xi \log X)^{n+1}} N(\mathfrak{A})^{-1} \tilde{m}(\mathfrak{A}, \gamma)
$$

Since

$$
\tilde{m}(\mathfrak{A}, \gamma)=\prod_{\mathfrak{p} \in \mathcal{P}, \mathfrak{p} \mid \mathfrak{A}}\left(1+N(\mathfrak{p})^{-1}\right)^{-1}
$$

when $b_{\mathfrak{A}} \neq 0$, one can conclude the proof of the lemma along the lines of $[3$, $\S 10 ; 4, \S 5]$.

As a consequence of Lemmata 3.1-3.3, Lemma 4.1, and the analogue of [4, Lemma 4.1], one obtains the following analogue of [4, Proposition 5.1].

Proposition 4.1 We have

$$
\begin{aligned}
\pi(\mathcal{A})-\kappa \pi(\mathcal{B}) & \ll \tau \frac{\eta^{2} X^{2}}{\log X}+\eta^{5 / 2} X^{2}(\log X)^{c} \\
& +\sum_{n \geq 3}\left|\hat{U}_{g}^{(n)}(\mathcal{A})\right|+\sum_{n=1,2}\left|\hat{U}_{1, g}^{(n)}(\mathcal{A})\right|+\left|\hat{S}_{4, g}(\mathcal{A})\right|+\left|\hat{U}_{2, g}^{(1)}(\mathcal{A})\right|
\end{aligned}
$$

As has been explained at the end of $[3, \S 3]$ and noted in [4, p.287], Theorem 2 and therefore Theorem 1 can be deduced from Proposition 4.1 and the following analogue of [4, Proposition 6.1].

Proposition 4.2 In the notation of $[4, \S 6]$, let $\mathcal{C} \subseteq \mathbb{R}^{3}$ be a cube of side $S_{0} \geq L^{2}$, and suppose that $c_{i} \ll V^{1 / 3}, 1 \leq i \leq 3$, and $N \check{c} \gg V$ for $\vec{c} \in \mathcal{C}$.

(i) There is a positive constant $c$ depending at most on $f$ and such that the following estimate

$$
\sum_{\substack{\beta=\alpha \bmod q \\ \hat{\beta} \in \mathcal{C}, \beta \in A^{(0)}}} g_{(\beta)} \ll V \exp (-c \sqrt{\log L})
$$

holds uniformly in a range $1<q \leq(\log X)^{l}$ for any $l>0$, any class $A$ of ideal numbers, and any ideal number $\alpha$ in $A$.

(ii) If a bound of the form

$$
\sum_{\substack{\beta=\alpha \bmod q \\ \hat{\beta} \in \mathcal{C}, \beta \in A^{(0)}}} g_{(\beta)} \ll V \exp (-c \sqrt{\log L}), c>0,
$$


holds uniformly in a range

$$
1<q \leq Q_{1} \leq \exp (\sqrt[3]{\log X})
$$

for any class $A$ of ideal numbers and any ideal number $\alpha$ in $A$, then

$$
\sum_{\substack{\mathfrak{a} \mathfrak{b} \in \mathcal{A} \\ V<N \mathfrak{b} \leq 2 V}} b_{\mathfrak{a}} g_{\mathfrak{b}} \ll X^{2} Q_{1}^{-1 / 160}(\log X)^{c}
$$

for $X^{1+\tau} \ll V \ll X^{3 / 2-\tau}$, with a suitable positive constant $c$ depending at most on $f$.

Only the second assertion (ii) needs a new proof. Choose $V$ as in (ii), and let

$$
\Sigma_{8}(V):=\sum_{\mathfrak{a} \mathfrak{b} \in \mathcal{A}, V<N \mathfrak{b} \leq 2 V} b_{\mathfrak{a}} g_{\mathfrak{b}}
$$

We have

$$
\Sigma_{8}(V)=\sum_{\substack{\mathfrak{a} \mathfrak{b}=\mathfrak{A}_{a}(\gamma), \vec{a} \in R \\ V<N \mathfrak{b} \leq 2 V}} b_{\mathfrak{a}} g_{\mathfrak{b}} \Psi(\vec{a})
$$

where $\Psi: \mathbb{R}^{2} \rightarrow\{0,1\}$ is the characteristic function of the square $I(X)$ and

$$
R:=\left\{\vec{a}: \vec{a} \in \mathbb{Z}^{2} \text {, h.c.f. }\left(\gamma_{1}+a_{1} d, \gamma_{2}+a_{2} d\right)=1\right\} .
$$

As in [4], let $\mathcal{Q}$ stand for the set of those integral ideals in $\mathfrak{o}$ which are not divisible by a rational prime; then

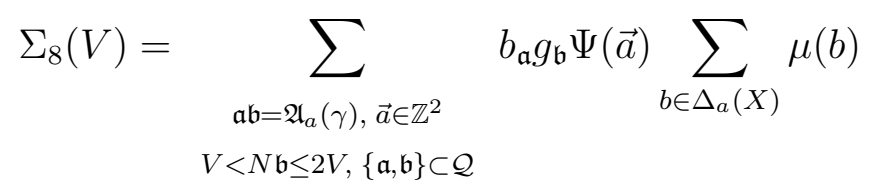

with

$$
\Delta_{a}(X):=\left\{b: b \in \mathbb{N}, b \ll X, b \mid \text { h.c.f. }\left(\gamma_{1}+a_{1} d, \gamma_{2}+a_{2} d\right)\right\} \text {. }
$$

Let $b \in \Delta_{a}(X)$. Then h.c.f. $(b, d)=1$ since, by assumption, h.c.f. $\left(\gamma_{1}, \gamma_{2}, d\right)=$ 1 ; therefore $b \mid \mathfrak{A}_{a}(\gamma)$. As in $[3, \S 11 ; 4, \S 6]$, it follows now that

$$
\Sigma_{8}(V)=\sum_{\substack{\mathfrak{a} \mathfrak{b}=\mathfrak{A}_{a}(\gamma),\{\mathfrak{a}, \mathfrak{b}\} \subset \mathcal{Q} \\ \vec{a} \in \mathbb{Z}^{2}, V<N \mathfrak{b} \leq 2 V}} b_{\mathfrak{a}} g_{\mathfrak{b}} \Psi(\vec{a})+O\left(X^{2-\tau / 2}(\log X)^{c}\right)
$$


for some $c$. In the notation of $[4, \S 6]$, equation (4.5) may be rewritten as follows:

$$
\Sigma_{8}(V)=\sum_{\substack{\varphi(\vec{a})=\delta \alpha \beta,(\alpha) \in \mathcal{Q} \\ \vec{a} \in \mathbb{Z}^{2}, V<N \beta \leq 2 V}} b_{(\alpha)} G(\beta) \Psi(\vec{a})+O\left(X^{2-\tau / 2}(\log X)^{c}\right),
$$

where now $\varphi(\vec{a}):=\gamma+d \cdot a$ and $\mathfrak{d}=(\delta)$. Proceeding as in [3, pp.67-68] and $[4$, p.279], we let

$$
\psi\left(\vec{a}_{1}, \vec{a}_{2} ; \beta_{1}, \beta_{2}\right)=\operatorname{card}\left\{\alpha:(\alpha) \in \mathcal{Q}, \varphi\left(\vec{a}_{i}\right)=\delta \alpha \beta_{i}, i=1,2\right\}
$$

and conclude that

$$
\Sigma_{8}(V) \ll X^{2-\tau / 2}(\log X)^{c}+\left(X^{3} / V\right)^{1 / 2} \Sigma_{9}^{1 / 2},
$$

where

$$
\Sigma_{9}=\Sigma_{10}+O\left(X^{2}(\log X)^{c}\right)
$$

with

$$
\Sigma_{10}:=\sum_{\substack{\beta_{1} \neq \beta_{2}, \vec{a}_{i} \in \mathbb{Z}^{2}, V<N \beta_{i} \leq 2 V, i=1,2}} G\left(\beta_{1}\right) G\left(\beta_{2}\right) \Psi\left(\vec{a}_{1}\right) \Psi\left(\vec{a}_{2}\right) \psi\left(\vec{a}_{1}, \vec{a}_{2} ; \beta_{1}, \beta_{2}\right) .
$$

For $\vec{b} \in \mathbb{Z}^{3}$, let

$$
D(\vec{b}):=\text { h.c.f. }\left(b_{1}, b_{2}, b_{3}\right),[\vec{b}]=D(\vec{b})^{-1} \vec{b}
$$

As in [4, p.279], one concludes that

$$
\psi\left(\vec{a}_{1}, \vec{a}_{2} ; \beta_{1}, \beta_{2}\right)=1 \Rightarrow \operatorname{cl} \beta_{1}=\operatorname{cl} \beta_{2} .
$$

Let us recall that, for each class $A$ of ideal numbers in $k$, we have defined [4, p.277] an invertible $\mathbb{Q}$-linear transformation

$$
h_{A}: \beta \mapsto \hat{\beta}, h_{A}: A \rightarrow \mathbb{Q}^{3}
$$

such that the image $h_{A}\left(A^{(0)}\right)$ of the lattice

$$
A^{(0)}=\{\alpha: \alpha \in A,(\alpha) \in \mathcal{I}(k)\}
$$

of the integral ideal numbers in $A$ is a sublattice of $\mathbb{Z}^{3}$ of finite index (equal to $\left|\operatorname{det} h_{A}\right|$ ). The following lemma is our analogue of [4, Lemma 6.2]. It can be deduced from relations (4.6)-(4.9) along the lines of $[3, \S 11 ; 4, \S 6]$. 
Lemma 4.2 Let $1 \ll Y \ll X^{\tau / 3}$. There is a constant $c$, depending at most on $f$ and a class $B$ of ideal numbers, such that

$$
\Sigma_{8}(V) \ll X^{2} Y^{-1 / 2}(\log X)^{c}+X^{3 / 2} V^{-1 / 2} \Sigma_{11}^{1 / 2}
$$

with

$$
\Sigma_{11}:=\sum_{\beta_{1}, \beta_{2}, \vec{a}_{1}, \vec{a}_{2}} G\left(\beta_{1}\right) G\left(\beta_{2}\right) \Psi\left(\vec{a}_{1}\right) \Psi\left(\vec{a}_{2}\right) \psi\left(\vec{a}_{1}, \vec{a}_{2} ; \beta_{1}, \beta_{2}\right)
$$

subject to the conditions

$$
D\left(\hat{\beta}_{1} \wedge \hat{\beta}_{2}\right)>V X^{-1} Y^{-1} ; \vec{a}_{i} \in \mathbb{Z}^{2}, \beta_{i} \in B^{(0)}, V<N \beta_{i} \leq 2 \text { for } i=1,2 .
$$

Moreover, if $\psi\left(\vec{a}_{1}, \vec{a}_{2} ; \beta_{1}, \beta_{2}\right)=1$, then

$$
\vec{a}_{i} d+\vec{\gamma}=\nu D\left(\hat{\beta}_{1} \wedge \hat{\beta}_{2}\right)^{-1} \vec{h}_{i}\left(\beta_{1}, \beta_{2}\right)
$$

with $\nu \in\{ \pm 1\}, \vec{h}_{i}:=\left(h_{i 1}, h_{i 2}\right)$, the functions $h_{i j}$ being defined by [4, (6.4)] for $i, j=1,2$.

Let us substitute relation (4.12) into the definition (4.11). This gives

$$
\Sigma_{11}=\sum_{\beta_{1}, \beta_{2}, \nu} G\left(\beta_{1}\right) G\left(\beta_{2}\right) \Psi\left(\frac{\nu D^{-1} \vec{h}_{1}\left(\beta_{1}, \beta_{2}\right)-\vec{\gamma}}{d}\right) \Psi\left(\frac{\nu D^{-1} \vec{h}_{2}\left(\beta_{1}, \beta_{2}\right)-\vec{\gamma}}{d}\right)
$$

subject to the conditions:

$$
D\left(\hat{\beta}_{1} \wedge \hat{\beta}_{2}\right)>V X^{-1} Y^{-1}
$$

and

$$
\beta_{i} \in B^{(0)}, V<N \beta_{i} \leq 2 V, \nu \vec{\gamma}=D^{-1} \vec{h}_{i}\left(\beta_{1}, \beta_{2}\right)(\bmod d)
$$

for $\nu \in\{ \pm 1\}, i=1,2$; here $D:=D\left(\hat{\beta}_{1} \wedge \hat{\beta}_{2}\right)$. Let

$$
\begin{aligned}
U(y, \nu) & =\left\{\left(\hat{\beta}_{1}, \hat{\beta}_{2}\right): \beta_{i} \in B^{(0)}, V<N \beta_{i} \leq 2 V,\right. \\
y\left(X d+\gamma_{j}\right) & \left.<\nu h_{i j}\left(\beta_{1}, \beta_{2}\right) \leq y\left(X(1+\eta) d+\gamma_{j}\right) \text { for } i, j=1,2\right\}
\end{aligned}
$$

for $y>0, \nu \in\{ \pm 1\}$; further, let

$$
\begin{aligned}
U_{0}(n, \nu) & =\left\{\left(\hat{\beta}_{1}, \hat{\beta}_{2}\right):\left(\hat{\beta}_{1}, \hat{\beta}_{2}\right) \in U(n, \nu),\right. \\
D\left(\hat{\beta}_{1} \wedge \hat{\beta}_{2}\right) & \left.=n, \nu \vec{\gamma}=n^{-1} \vec{h}_{i}\left(\beta_{1}, \beta_{2}\right)(\bmod d) \text { for } i=1,2\right\}
\end{aligned}
$$


for $n \in \mathbb{N}$. As in [3, p.72; 4, p.280], let us subdivide the range of summation in $\Sigma_{11}$ into subintervals

$$
I_{m}:=\left(\frac{m-1}{T} \Delta, \frac{m}{T} \Delta\right], \text { with } T \ll X^{2 \tau / 3}, T<m \leq 2 T, \Delta \ll V X^{-1} .
$$

Let

$$
\Sigma_{12}(m, \Delta)=\sum_{d_{1} \in I_{m}}\left|\sum_{\left(\hat{\beta_{1}}, \hat{\beta_{2}}\right) \in U_{0}\left(d_{1}, \nu\right)} G\left(\beta_{1}\right) G\left(\beta_{2}\right)\right| .
$$

As in the cited works (cf. [4, (6.6), (6.7)]), it follows from equation (4.13) that

$$
\Sigma_{11} \ll(\log X) T \Sigma_{12}(m, \Delta),
$$

for at least one triple $(m, \Delta, \nu)$. Proceeding with our argument, let us cover the region of summation $U_{0}\left(d_{1}, \nu\right)$ in (4.14) by means of cubes $\mathfrak{C}(\vec{n})=\mathcal{C}_{1}(\vec{n}) \times$ $\mathcal{C}_{2}(\vec{n})$ defined as in $[4, \S 6]$. Relations $(4.10),(4.14-16)$ lead to the following inequality (cf. [3, (12.8); 4, (6.9)]):

$$
\Sigma_{8}(V) \ll X^{2} Y^{-1 / 2}(\log X)^{c}+X^{3 / 2} V^{-1 / 2} Y^{7} \Sigma_{13}^{1 / 2}(\log X)^{c}
$$

with

$$
\Sigma_{13}=\sum_{d_{1} \in I_{m}}\left|\sum_{\left(\hat{\beta_{1}}, \hat{\beta_{2}}\right) \in U_{1}\left(\mathfrak{C} ; d_{1}, \nu\right)} G\left(\beta_{1}\right) G\left(\beta_{2}\right)\right|,
$$

where

$$
\begin{aligned}
U_{1}\left(\mathfrak{C}_{i} d_{1}, \nu\right) & =\left\{\left(\hat{\beta}_{1}, \hat{\beta}_{2}\right):\left(\hat{\beta}_{1}, \hat{\beta}_{2}\right) \in \mathfrak{C}, D\left(\hat{\beta}_{1} \wedge \hat{\beta}_{2}\right)=d_{1},\right. \\
\beta_{i} \in B^{(0)}, \nu \vec{\gamma} & \left.=d_{1}^{-1} \vec{h}_{i}\left(\beta_{1}, \beta_{2}\right)(\bmod d) \text { for } i=1,2\right\}
\end{aligned}
$$

and $\mathfrak{C}$ is a suitable class I cube, in the sense of [3, p.73; 4, p.281]. Let

$$
\begin{aligned}
U_{2}\left(\mathfrak{C} ; d_{1}, d_{2}, \nu\right) & =\left\{\left(\hat{\beta}_{1}, \hat{\beta}_{2}\right):\left(\hat{\beta}_{1}, \hat{\beta}_{2}\right) \in \mathfrak{C}, d_{1} d_{2} \mid \hat{\beta}_{1} \wedge \hat{\beta}_{2},\right. \\
\beta_{i} \in B^{(0)}, \nu \vec{\gamma} & \left.=d_{1}^{-1} \vec{h}_{i}\left(\beta_{1}, \beta_{2}\right)(\bmod d) \text { for } i=1,2\right\}
\end{aligned}
$$

and let

$$
\Sigma_{14}=\sum_{\left(\hat{\beta}_{1}, \hat{\beta}_{2}\right) \in U_{2}\left(\mathfrak{C} ; d_{1}, d_{2}, \nu\right)} G\left(\beta_{1}\right) G\left(\beta_{2}\right) .
$$

The argument used in $[3, \S 12]$ and [4, pp.281-283] to deduce [3, Lemma 12.2] and its analogue $[4,(6.11)]$ leads to the following conclusion.

Lemma 4.3 Suppose that $1 \ll Y \ll X^{\tau / 3}$, and let $d_{0}:=X^{-1} V Y^{15}+V^{1 / 6}$. There is a constant $c$, depending at most on $f$, such that

$$
\Sigma_{8}(V) \ll X^{2} Y^{-1 / 2}(\log X)^{c}+X^{3 / 2} V^{-1 / 2} Y^{7} \Sigma_{15}^{1 / 2}(\log X)^{c},
$$

where

$$
\Sigma_{15}=\sum_{d_{1} \in I_{m}, d_{1} d_{2}<d_{0}}\left|\Sigma_{14}\right|
$$

and $\mathfrak{C}$ is a suitable class I cube. 


\section{Completion of the proof of Proposition 4.2 and Theorem 2}

The functions

$$
\vec{h}_{i}\left(\beta_{1}, \beta_{2}\right)=\vec{h}_{i}\left(h_{B}^{-1}\left(\hat{\beta}_{1}\right), h_{B}^{-1}\left(\hat{\beta}_{2}\right)\right)
$$

may be regarded as polynomials in $\hat{\beta}_{1}$ and $\hat{\beta}_{2}$ with rational coefficients. We write them with a common denominator as

$$
\vec{h}_{i}\left(\beta_{1}, \beta_{2}\right)=d^{*-1} \vec{H}_{i}\left(\hat{\beta}_{1}, \hat{\beta}_{2}\right)
$$

where each $\vec{H}_{i}$ is a vector of three integral polynomials. We note that the common denominator $d^{*}$ will only depend on the field $k$ and the choice of the bases made in [4, p. 277]. Moreover, we note from the definition [4; $(6.4)]$ that $\vec{h}_{i}(\beta, \lambda \beta)$ vanishes identically for $i=1,2$, whence

$$
\vec{H}_{i}(\hat{\beta}, \lambda \hat{\beta})=0 \quad(i=1,2)
$$

identically.

The conditions

$$
\nu \vec{\gamma}=d_{1}^{-1} \vec{h}_{i}\left(\beta_{1}, \beta_{2}\right)(\bmod d) \text { for } i=1,2
$$

are now equivalent to a pair of congruences

$$
d_{1} d^{*} \nu \vec{\gamma}=\vec{H}_{i}\left(\hat{\beta}_{1}, \hat{\beta}_{2}\right)\left(\bmod d_{1} d d^{*}\right) .
$$

In order to separate the variables in the equation (4.17), let us replace the condition $d_{1} d_{2} \mid \hat{\beta}_{1} \wedge \hat{\beta}_{2}$ by the condition that $\hat{\beta}_{2}=\lambda \hat{\beta}_{1}\left(\bmod d_{1} d_{2}\right)$ for some integer $\lambda$, which is necessarily coprime to $d_{1} d_{2}$, cf. [3, pp. 70, 78]; it may clearly be assumed that $1 \leq \lambda \leq d_{1} d_{2}$.

Since our conditions are now all modulo $d_{12}:=d_{1} d_{2} d d^{*}$, we can pick out the admissible vectors $\hat{\beta}_{i}$ by using orthogonality for the function

$$
e_{l}(x):=\exp (2 \pi i x / l)
$$

and taking $l=d_{12}$. In this way we find that

$$
\Sigma_{14}=\frac{1}{d_{12}^{6}} \sum_{\lambda, \beta_{i}, \vec{b}_{i}, \hat{\eta}_{i}} e_{d_{12}}\left(\vec{b}_{1}\left(\hat{\beta}_{1}-\hat{\eta}_{1}\right)+\vec{b}_{2}\left(\hat{\beta}_{2}-\hat{\eta}_{2}\right)\right) G\left(\beta_{1}\right) G\left(\beta_{2}\right)
$$

subject to the conditions:

$$
\hat{\eta}_{2}=\lambda \hat{\eta}_{1}\left(\bmod d_{1} d_{2}\right), 1 \leq \lambda \leq d_{1} d_{2},\left(\lambda, d_{1} d_{2}\right)=1,0 \leq b_{i j}, \hat{\eta}_{i j}<d_{12},
$$




$$
d_{1} d^{*} \nu \vec{\gamma}=\vec{H}_{i}\left(\hat{\eta}_{1}, \hat{\eta}_{2}\right)\left(\bmod d_{1} d d^{*}\right), \beta_{i} \in B^{(0)}, \hat{\beta}_{i} \in \mathcal{C}_{i},
$$

where $\mathfrak{C}=\mathcal{C}_{1} \times \mathcal{C}_{2}, \hat{\eta}_{i}=\left(\hat{\eta}_{i 1}, \hat{\eta}_{i 2}, \hat{\eta}_{i 3}\right)$, for $1 \leq j \leq 3$ and $i=1,2$. Given $\mathcal{D} \subset \mathbb{R}^{3}$ and $\alpha \in \mathbb{R}^{3}$, let

$$
\sigma_{l}(\alpha, \mathcal{D})=\sum_{\hat{\beta} \in \mathcal{D} \cap \mathbb{Z}^{3}, \beta \in B^{(0)}} e_{l}(\alpha \hat{\beta}) G(\beta),
$$

whence

$$
\Sigma_{14}=\frac{1}{d_{12}^{6}} \sum_{\lambda, \vec{b}_{i}, \hat{\eta}_{i}} e_{d_{12}}\left(-\vec{b}_{1} \hat{\eta}_{1}-\vec{b}_{2} \hat{\eta}_{2}\right) \sigma_{d_{12}}\left(\vec{b}_{1}, \mathcal{C}_{1}\right) \sigma_{d_{12}}\left(\vec{b}_{2}, \mathcal{C}_{2}\right)
$$

with $\lambda, \hat{\eta_{1}}, \hat{\eta_{2}}$ subject to the same conditions as before. We proceed to write $\hat{\eta}_{2}=\lambda \hat{\eta}_{1}+d_{1} d_{2} \hat{\eta}_{3}$, where $\hat{\eta}_{3}$ runs over integer vectors modulo $d d^{*}$ for which

$$
d_{1} d^{*} \nu \vec{\gamma}=\vec{H}_{i}\left(\hat{\eta}_{1}, \lambda \hat{\eta}_{1}+d_{1} d_{2} \hat{\eta}_{3}\right)\left(\bmod d_{1} d d^{*}\right) .
$$

According to (5.1) the right hand side depends only on the congruence classes of $\hat{\eta}_{1}$ and $\hat{\eta_{3}}$ modulo $d d^{*}$. We therefore put $\hat{\eta}_{1}=\hat{\eta_{4}}+d d^{*} \hat{\eta}_{5}$, where $\hat{\eta_{4 i}}$ runs over integer vectors modulo $d d^{*}$ and $\hat{\eta}_{5 i}$ runs over integer vectors modulo $d_{1} d_{2}$. The sum $\Sigma_{14}$ now becomes

$$
\Sigma_{14}=\frac{1}{d_{12}^{6}} \sum_{\lambda, \vec{b}_{i}} S\left(\lambda, \vec{b}_{1}, \vec{b}_{2}\right) \sigma_{d_{12}}\left(\vec{b}_{1}, \mathcal{C}_{1}\right) \sigma_{d_{12}}\left(\vec{b}_{2}, \mathcal{C}_{2}\right)
$$

with $\overrightarrow{b_{1}}, \overrightarrow{b_{2}}$ and $\lambda$ running over the same set as before, and

$$
S\left(\lambda, \overrightarrow{b_{1}}, \vec{b}_{2}\right)=\sum_{\hat{\eta_{3}}, \hat{\eta_{4}}, \hat{\eta_{5}}} e_{d_{1} d_{2}}\left(-\left(\overrightarrow{b_{1}}+\lambda \overrightarrow{b_{2}}\right) \hat{\eta_{5}}\right) e_{d_{12}}\left(-\left(\overrightarrow{b_{1}}+\lambda \overrightarrow{b_{2}}\right) \hat{\eta}_{4}-d_{1} d_{2} \vec{b}_{2} \hat{\eta_{3}}\right)
$$

with

$$
0 \leq \hat{\eta_{3 i}}<d d^{*}, \quad 0 \leq \hat{\eta_{4 i}}<d d^{*}, \quad 0 \leq \hat{\eta_{5 i}}<d_{1} d_{2} \text { for } 1 \leq i \leq 3,
$$

subject to the condition

$$
d_{1} d^{*} \nu \vec{\gamma}=\vec{H}_{i}\left(\hat{\eta}_{4}, \lambda \hat{\eta}_{4}+d_{1} d_{2} \hat{\eta}_{3} d_{1} d d^{*}\right)\left(\bmod d_{1} d d^{*}\right) .
$$

We may therefore perform the summation over $\hat{\eta}_{5}$ to deduce that

$$
S\left(\lambda, \vec{b}_{1}, \vec{b}_{2}\right)=0
$$

unless

$$
\overrightarrow{b_{1}}+\lambda \overrightarrow{b_{2}}=0\left(\bmod d_{1} d_{2}\right)
$$


in which case we have the trivial bound

$$
S\left(\lambda, \vec{b}_{1}, \vec{b}_{2}\right) \ll\left(d_{1} d_{2}\right)^{3},
$$

on recalling that $d_{12} \ll d_{1} d_{2}$.

We now deduce from (5.2) that

$$
\Sigma_{14} \ll\left(d_{1} d_{2}\right)^{-3} \sum_{\lambda, \vec{b}_{i}}\left|\sigma_{d_{12}}\left(\vec{b}_{1}, \mathcal{C}_{1}\right) \sigma_{d_{12}}\left(\vec{b}_{2}, \mathcal{C}_{2}\right)\right|,
$$

where the sum is now subject to the condition

$$
1 \leq \lambda \leq d_{1} d_{2},\left(\lambda, d_{1} d_{2}\right)=1,0 \leq b_{i j}<d_{12}
$$

and

$$
\overrightarrow{b_{1}}+\lambda \overrightarrow{b_{2}}=0\left(\bmod d_{1} d_{2}\right)
$$

An application of Cauchy's inequality then yields

$$
\Sigma_{14} \ll\left(d_{1} d_{2}\right)^{-3}\left\{\sum_{\vec{b}_{1}}\left|\sigma_{d_{12}}\left(\vec{b}_{1}, \mathcal{C}_{1}\right)\right|^{2} N_{1}\right\}^{1 / 2}\left\{\sum_{\vec{b}_{2}}\left|\sigma_{d_{12}}\left(\vec{b}_{2}, \mathcal{C}_{2}\right)\right|^{2} N_{2}\right\}^{1 / 2}
$$

where $N_{1}$ is the number of pairs $\lambda, \overrightarrow{b_{2}}$ which can correspond to a given vector $\overrightarrow{b_{1}}$, and similarly for $N_{2}$. Clearly we have $N_{1}, N_{2} \ll d_{1} d_{2}$, and it therefore follows that

$$
\Sigma_{14} \ll\left(d_{1} d_{2}\right)^{-2} \max _{i=1,2} \sum_{\vec{b}}\left|\sigma_{d_{12}}\left(\vec{b}, \mathcal{C}_{i}\right)\right|^{2}
$$

where $\vec{b}$ runs over integer vectors modulo $d_{12}$.

We then have

$$
\Sigma_{14} \ll\left(d_{1} d_{2}\right)^{-2}\left\{\sum_{\vec{b}}\left|\sigma_{d_{12}}\left(\vec{b}, \mathcal{C}_{1}\right)\right|^{2}+\sum_{\vec{b}}\left|\sigma_{d_{12}}\left(\vec{b}, \mathcal{C}_{2}\right)\right|^{2}\right\},
$$

which, in conjunction with (4.17), produces the upper bound

$$
\Sigma_{15} \ll \sum_{\substack{d_{1} \in I_{m}, d_{1} d_{2}<d_{0} \\ \vec{b} \bmod d_{12}}} \frac{1}{\left(d_{1} d_{2}\right)^{2}}\left|\sigma_{d_{12}}\left(\vec{b}, \mathcal{C}_{0}\right)\right|^{2}
$$

with $\mathcal{C}_{0} \in\left\{\mathcal{C}_{1}, \mathcal{C}_{2}\right\}$, cf. $[3$, p.78; 4, p.283]. Proposition 4.2 can be deduced from Lemma 4.3 and relation (5.3) along the lines of $[3, \S 13 ; 4, \S 6]$.

On suitably adjusting the parameters $\eta$ and $Q_{1}$, [3, p.21; 4, p.287], one concludes the proof of Theorems 1 and 2 as in the cited works. 


\section{References}

[1] H. Halberstam and H.-E. Richert, Sieve methods, Academic Press, London, 1974.

[2] D.R. Heath-Brown, The solubility of diagonal cubic Diophantine equations, Proceedings of the London Mathematical Society (3), 79 (1999), 241-259.

[3] D.R. Heath-Brown, Primes represented by $x^{3}+2 y^{3}$, Acta Mathematica, 186 (2001), 1-84.

[4] D.R. Heath-Brown and B.Z. Moroz, Primes represented by binary cubic forms, Proceedings of the London Mathematical Society (3), 84 (2002), 257-288.

[5] P. Llorente and E. Nart, Effective determination of the decomposition of the rational primes in a cubic field, Proc. of the American Math. Soc., 87 (1983), 579-585.

[6] P. Satgé, Un analogue du calcul de Heegner, Invent. Math., 87 (1987), 425-439.

[7] H.Weyl, Algebraic theory of numbers, Princeton University Press, Princeton, 1940. 